PROCEEDINGS OF THE

AMERICAN MATHEMATICAL SOCIETY

Volume 126, Number 6, June 1998, Pages 1799-1810

S 0002-9939(98)04726-1

\title{
ON REPRESENTATION AND REGULARITY OF CONTINUOUS PARAMETER MULTIVALUED MARTINGALES
}

\author{
DONG WENLONG AND WANG ZHENPENG
}

(Communicated by Richard T. Durrett)

\begin{abstract}
In this paper we study multivalued martingales in continuous time. First we show that every multivalued martingale in continuous time can be represented as the closure of a sequence of martingale selections. Then we prove two results concerning the cadlag modifications of continuous time multivalued martingales, in Kuratowski-Mosco convergence and in convergence in the Hausdorff metric respectively.
\end{abstract}

\section{$\S 1$. INTRODUCTION}

Multivalued processes (set-valued processes) in discrete time have been studied by many authors. This notion extends those of real and vector random processes, and it is interesting that there are a lot of convergence and representation results analogous to those existing for single-valued processes. This line of recent research can be traced in the works of Bagchi [1, 2], Dam [9], Daures [10], Giné-Hahn-Zinn [15], Gut-Schmidt [16], Hess [17, 18], Hiai-Umegaki [20], Luu [22], Papageorgiou [25]-[29], Salinetti-Wets [31] and Wang-Xue [34]. The more recent works among this list obtained many profound convergence results and representation theorems concerning martingale or amart selections for multivalued martingale-like processes.

As for the study of the regularity of vector-valued processes which admit cadlag modifications, we can refer to the works of Choi-Sucheston [8] concerning uniform amarts and Frangos [13] concerning separable positive integrable submartingales on an ordered continuous Banach lattice.

The purpose of the present paper is to discuss some questions of continuous parameter multivalued martingales. The questions are their representations and their cadlag modifications. Some preliminaries are given in Section 2. In Section 3, a representation result for closed martingales is given; the main result of this section is the representation theorem for continuous parameter multivalued martingales which have weakly compact and convex values or whose dual space $\mathbf{X}^{*}$ is strongly separable. In Section 4, a general limit theorem analogous to Theorems 2.3 and 3.2 of Choi-Sucheston [8] is given first. Then we prove two convergence results for closed martingales in the ascending and descending cases in Kuratowski-Mosco (K-M) convergence and in convergence in the Hausdorff metric. Finally, Section 5

Received by the editors May 4, 1995 and, in revised form, May 12, 1996.

1991 Mathematics Subject Classification. Primary 60D05, 60G25, 60G35, 60G48.

Key words and phrases. Cadlag modification, continuous parameter multivalued martingales, Kuratowski-Mosco convergence, martingale selections, right-closed martingales.

(C)1998 American Mathematical Society 
provides two other main results concerning the cadlag modificatons for multivalued martingales in continous time. Theorem 5.3 admits the right continuity and the left-limit in the cadlag modification with respect to K-M convergence, and every such process is uniformly bounded (for $t \geq 0$ ) by a multifunction which has weakly compact and convex values. Then Theorem 5.4 gives convergence in the Hausdorff metric under the restriction that these processes can be obtained as the limit of simple multifunctions in the complete metric space $\left(\mathcal{L}_{f c}^{1}(\mathbf{X}), \Delta\right)$.

\section{§2. Notations AND PRELIMINARIES}

Throughout this paper, $(\Omega, \mathcal{F}, \mathbf{P})$ is a complete probability space. We denote by $\mathbf{Z}$ (resp. J,,$\overline{\mathbf{J}}$ ) the set of (resp. positive, nonnegative) integers and by $\mathbf{R}$ (resp. $\mathbf{R}_{+}$) the set of real numbers (resp. nonnegative real numbers). Let $\left(\mathcal{F}_{t}, t \geq 0\right)$ be a sub $\sigma$-field filtration on $(\Omega, \mathcal{F}, \mathbf{P})$, which satisfies the usual conditions (complete and right continuity). $\tau$ is a stopping time of $\left(\mathcal{F}_{t}\right)$. An increasing sequence $\tau_{1}, \tau_{2}$, ... of stopping times is said to announce $\tau$ if $\lim _{n \rightarrow \infty} \tau_{n}=\tau$ and $\tau_{n}<\tau$ (except on $\{\tau=0\})$. Similarily a decreasing sequence of stopping times is said to recall $\tau$ if $\lim _{n \rightarrow \infty} \tau_{n}=\tau$ and $\tau_{n}>\tau$ (except on $\{\tau=\infty\}$ ). A predictable time is a stopping time that is announced by some sequence $\left(\tau_{n}\right)$. A stopping time is called simple if it takes finitely many finite values. Let $\mathbf{T}$ denote the set of all simple stopping times and $\mathbf{T}(\mathbf{S})$ the set of all simple stopping times with values in a countable dense subset $\mathbf{S}$ of $\mathbf{R}_{+}$containing 0 .

$\mathbf{X}$ denotes a separable Banach space with the dual space $\mathbf{X}^{*}, \mathcal{C}$ the family of nonempty bounded close convex subsets of $\mathbf{X}, \mathcal{K}$ the family of nonempty weakly compact convex subset of $\mathbf{X}$. We denote by $\mathbf{D}$ a countable dense subset of $\mathbf{X}$ and by $\mathbf{D}^{*}$ (resp. $\mathbf{D}^{*}(M)$ ) a countable dense subset of $\mathbf{X}^{*}$ in strong topology (resp. the Mackey topology $m\left(\mathbf{X}^{*}, \mathbf{X}\right)$ ). Given subsets $A, B$ and $C$ of $\mathbf{X}$, the distance function $d(\cdot, C)$, the support function $s(\cdot, C)$ and the Hausdorff distance $h(A, B)$ are defined as in Hiai-Umegaki [20], Hess [17] and Wang-Xue [34]. Let $\mathcal{G}$ be a sub $\sigma$-field of $\mathcal{F}$; the concepts and notations such as $\mathbf{X}$-valued Bochner integrable random variables space $L^{1}[\Omega, \mathbf{X}]$, measurable multifunctions $\mathcal{M}[\mathcal{F}]$, Castaing representations, integrable $\mathcal{C}$-valued multifunctions space $\mathcal{L}_{\text {fc }}^{1}$, integrable compact $\mathcal{C}$ valued multifunctions $\mathcal{L}_{c c}^{1}$, integrable $\mathcal{K}$-valued multifunctions $\mathcal{L}_{w k c}^{1}, \mathcal{G}$-measurable integral set $S_{F}^{1}(\mathcal{G})$ of multifunction $F$, conditional expectations, etc. are the same as in the above references too.

Let $\mathbf{Q}$ be a directed index subset of $\mathbf{R}$ containing infinity, $t \in \mathbf{Q}$, and let $\left(C_{r}\right)_{r \in \mathbf{Q}}$ be a class of subsets of $\mathbf{X}$. We put

$$
\begin{aligned}
s-\varliminf_{s \rightarrow t} C_{s}:= & \left\{x \in \mathbf{X} \mid x=\lim _{s \rightarrow t} x_{s}, x_{s} \in C_{s}, \forall s \in \mathbf{Q}\right\}, \\
w-\varlimsup & \varlimsup C_{s}:= \\
& \left\{x \in \mathbf{X} \mid\left(x_{k}\right) \text { is weakly convergent to } x,\right. \\
& \left.x_{k} \in C_{s(k)}, \forall k \in \mathbf{J}\right\}
\end{aligned}
$$

where $\left(C_{s(k)}\right)_{k \in J}$ is a subsequence of $\left(C_{s}\right)_{s \in \mathbf{Q}}$ and $s(k) \rightarrow t$. A directed class $\left(C_{r}\right)$ is said to converge to $C$ in the sense of Kuratowski-Mosco $(K-M)$, if

$$
C=s-\varliminf_{s \rightarrow t} C_{s}=w-\varlimsup_{s \rightarrow t} C_{s} .
$$

We write this limit as $(\mathrm{K}-\mathrm{M}) \lim _{s \rightarrow t} C_{s}$ or $C_{s} \stackrel{\mathrm{K}-\mathrm{M}}{\longrightarrow} C$. We say it converges to $C$ in the sense of Wijsman, if for any $x \in \mathbf{X}, \lim _{s \rightarrow t} d\left(x, C_{s}\right)=d(x, C)$, that it weakly converges to $C$, if for any $x^{*} \in \mathbf{X}^{*}, \lim _{s \rightarrow t} s\left(x^{*}, C_{s}\right)=s\left(x^{*}, C\right)$, and that 
the Hausdorff distance converges to $C$, if $\lim _{s \rightarrow t} h\left(C_{s}, C\right)=0$. We denote the above limits by $C_{s} \stackrel{\text { Wijs }}{\longrightarrow} C, C_{s} \stackrel{w}{\longrightarrow} C$ and $C_{s} \stackrel{h}{\longrightarrow} C$ respectively. The relations among the above four kinds of convergence were mentioned in Wang-Xue [34, p.809].

For $F, G \in \mathcal{L}_{f c}^{1}$, define $\Delta(F, G)=\mathbf{E} h(F, G)$; then $(\mathcal{C}, h)$ and $\left(\mathcal{L}_{f c}^{1}, \Delta\right)$ are complete metric spaces. Let $\mathbf{L}_{c}^{1}$ be the closure of the set of all simple functions in $\left(\mathcal{L}_{f c}^{1}, \Delta\right)$ (cf. Hiai-Umegaki $[20$, p.160]). When $\mathbf{X}$ is a finite dimensional space, $\mathbf{L}_{c}^{1}=\mathcal{L}_{f c}^{1}$.

Definition 2.1. An adapted $\mathcal{L}_{f c}^{1}$-valued process $\left\{F_{t}, \mathcal{F}_{t}, t \in \mathbf{Q}\right\}$ (i.e., for every $t \in \mathbf{Q}$, the multifunction $F_{t}$ is $\mathcal{F}_{t}$-measurable) is called a (multivalued) martingale, submartingale, or supermartingale, if

$$
\mathbf{E}\left(F_{t} \mid \mathcal{F}_{s}\right)=, \supset \text {, or } \subset F_{s} \quad \text { a.s. }
$$

for all $s, t \in \mathbf{Q}, s<t$.

Definition 2.2. An adapted $\mathbf{X}$-valued process $\left\{f_{t}, \mathcal{F}_{t}, t \in \mathbf{Q}\right\}$ is called a martingale selection of an adapted multivalued process $\left\{F_{t}, \mathcal{F}_{t}, t \in \mathbf{Q}\right\}$ if it satisfies the following two conditions:

1. $\left\{f_{t}, \mathcal{F}_{t}, t \in \mathbf{Q}\right\}$ is a vector-valued martingale,

2. $\forall t \in \mathbf{Q}, f_{t} \in S_{F_{t}}^{1}\left(\mathcal{F}_{t}\right)$.

The set of martingale selections of a multivalued process $\left\{F_{t}, \mathcal{F}_{t}, t \in \mathbf{Q}\right\}$ will be denoted by $\operatorname{MS}\left(F_{t}\right)$ (or $\operatorname{MS}\left(F_{n}\right)$, if $\left.\mathbf{Q} \subset \mathbf{Z}\right)$.

We start with the following two simple lemmas.

Lemma 2.1. Suppose that $\left(C, C_{n}, n \in \mathbf{J}\right) \subset \mathcal{K}$ and $C \cup\left(\bigcup_{n \geq 1} C_{n}\right) \subset B \in \mathcal{K}$. Then

1. $C_{n} \stackrel{\text { Wijs }}{\longrightarrow} C$ if and only if

$$
\lim _{n \rightarrow \infty} d\left(x, C_{n}\right)=d(x, C), \quad \forall x \in \mathbf{D} .
$$

2. $C_{n} \stackrel{w}{\longrightarrow} C$ if and only if

$$
\lim _{n \rightarrow \infty} s\left(x^{*}, C_{n}\right)=s\left(x^{*}, C\right), \quad \forall x^{*} \in \mathbf{D}^{*}(M) .
$$

Thus we have the following lemma directly from Hess [17, Lemma 5.4]

Lemma 2.2. Under the conditions in Lemma 2.1, the following are equivalent:

1. $C_{n} \stackrel{\mathrm{K}-\mathrm{M}}{\longrightarrow} C$,

2. $\begin{cases}\lim _{n \rightarrow \infty} d\left(x, C_{n}\right)=d(x, C), & \forall x \in \mathbf{D}, \\ \lim _{n \rightarrow \infty} s\left(x^{*}, C_{n}\right)=s\left(x^{*}, C\right), & \forall x^{*} \in \mathbf{D}^{*}(M) .\end{cases}$

Now we define a useful distance on $\mathcal{K}$ to make it a metric space. Let $\mathbf{D}=\left(x_{k}, k \in\right.$ $\mathbf{J})$ and $\mathbf{D}^{*}(M)=\left(x_{k}^{*}, k \in \mathbf{J}\right)$. For any $A, B \in \mathcal{K}$

$$
\rho(A, B):=\sum_{k=1}^{\infty} \frac{1}{2^{k}}\left(\frac{\left|d\left(x_{k}, A\right)-d\left(x_{k}, B\right)\right|}{1+\left|d\left(x_{k}, A\right)-d\left(x_{k}, B\right)\right|}+\frac{\left|s\left(x_{k}^{*}, A\right)-s\left(x_{k}^{*}, B\right)\right|}{1+\left|s\left(x_{k}^{*}, A\right)-s\left(x_{k}^{*}, B\right)\right|}\right)
$$

$(\mathcal{K}, \rho)$ is a metric space. Under the same hypothesis as in Lemma 2.1, we have the following equivalence by Lemma 2.2:

$$
C_{n} \stackrel{\mathrm{K}-\mathrm{M}}{\longrightarrow} C \quad \Longleftrightarrow \quad \rho\left(C_{n}, C\right) \rightarrow 0
$$




\section{§3. Martingale selections and Representation theorems}

In this section we establish the representation theorem for continuous parameter multivalued martingales: Every multivalued martingale can be represented as the closure of a sequence of its martingale selections. Luu [22] obtained it for $\mathcal{L}_{f c^{-}}^{1}$ valued martingales with discrete parameter. Hess [17] generalized the result to martingales with unbounded values.

Let $\left(\mathcal{F}_{n}, n \in \mathbf{Z}\right)$ be an increasing $\sigma$-field filtration, $\mathcal{F}_{\infty}=\bigvee_{n \geq 1} \mathcal{F}_{n}, \mathcal{F}_{-\infty}=$ $\bigcap_{n<-1} \mathcal{F}_{n}$. First we give a simple extension of Proposition 1.4 in Luu [22] to closed multivalued martingales. We begin with the following lemma.

Lemma 3.1. Suppose that $F \in \mathcal{L}_{\text {fc }}^{1}$ and the $\sigma$-field $\mathcal{G} \subset \mathcal{F}$. Then there exists a sequence $\left\{f^{i}, i \geq 1\right\} \subset S_{F}^{1}$ such that $F(\omega)=\operatorname{cl}\left\{f^{i}(\omega) ; i \geq 1\right\}, \forall \omega \in \Omega$, and $\mathbf{E}(F \mid \mathcal{G})=\operatorname{cl}\left\{\mathbf{E}\left(f^{i} \mid \mathcal{G}\right) ; i \geq 1\right\}$, a.s.

Proof. There exist $\left(f^{n}, n \geq 1\right) \subset S_{F}^{1}$ and $\left(g^{n}, n \geq 1\right) \subset S_{\mathbf{E}(F \mid \mathcal{G})}^{1}(\mathcal{G})=\operatorname{cl}\{\mathbf{E}(f \mid \mathcal{G}) ; f \in$ $\left.S_{F}^{1}\right\}$ such that

$$
\begin{aligned}
& F(\omega)=\operatorname{cl}\left\{f^{n}(\omega) ; n \geq 1\right\}, \quad \text { a.s. } \\
& \mathbf{E}(F \mid \mathcal{G})(\omega)=\operatorname{cl}\left\{g^{n}(\omega) ; n \geq 1\right\}, \quad \text { a.s. }
\end{aligned}
$$

For any positive integer $m$, there exists a sequence $\left(f^{m, k}, k \geq 1\right) \subset S_{F}^{1}$ such that $\left\|g^{m}-\mathbf{E}\left(f^{m, k} \mid \mathcal{G}\right)\right\|_{1} \rightarrow 0(k \rightarrow \infty)$. Thus there exists a subsequence $(k(j), j \geq 1)$ such that $\left\|g^{m}-\mathbf{E}\left(f^{m, k(j)} \mid \mathcal{G}\right)\right\| \rightarrow 0$ almost surely, from which we have

$$
\mathbf{E}(F \mid \mathcal{G})=\operatorname{cl}\left\{\mathbf{E}\left(f^{m, k(j)} \mid \mathcal{G}\right) ; m \geq 1, j \geq 1\right\}, \quad \text { a.s. }
$$

Moreover, there exists a null set $N \in \mathcal{F}$ such that for all $\omega \in N^{c}, f^{m, k(j)}(\omega) \in$ $F(\omega), \forall m \geq 1, j \geq 1$. Let

$$
f^{m, k(j)}(\omega)= \begin{cases}f^{m, k(j)}(\omega) & \omega \in N^{c} \\ f^{1}(\omega) & \omega \in N .\end{cases}
$$

for $m \geq 1$ and $j \geq 1$. Then we have

$$
\begin{aligned}
& F=c l\left\{f^{n}, f^{m, k(j)} ; n \geq 1, m \geq 1, j \geq 1\right\} \\
& \mathbf{E}(F \mid \mathcal{G})=c l\left\{\mathbf{E}\left(f^{n} \mid \mathcal{G}\right), \mathbf{E}\left(f^{m, k(j)} \mid \mathcal{G}\right) ; n \geq 1, m \geq 1, j \geq 1\right\} \quad \text { a.s. }
\end{aligned}
$$

This completes the proof of Lemma 3.1.

From Lemma 3.1 we can easily prove the following theorem.

Theorem 3.1. Suppose that $F \in \mathcal{L}_{\text {fc }}^{1}$. Let $F_{n}=\mathbf{E}\left(F \mid \mathcal{F}_{n}\right),-\infty \leq n \leq+\infty$. Then there exists a sequence $\left(g^{i}, i \geq 1\right) \subset S_{F}^{1}$ such that $F=\operatorname{cl}\left\{g^{i} ; i \geq 1\right\}$ and

$$
F_{n}=\operatorname{cl}\left\{\mathbf{E}\left(g^{i} \mid \mathcal{F}_{n}\right) ; i \geq 1\right\} \quad \text { a.s., }-\infty \leq n \leq+\infty .
$$

In the sequel, we prove the representation theorem for continuous parameter multivalued martingales.

Lemma 3.2. If $F, G \in \mathcal{L}_{w k c}^{1}$ satisy $F \subset G$ a.s. and $\mathbf{E}(F)=\mathbf{E}(G)$, then $F=G$, a.s.

Proof. For all $x^{*} \in \mathbf{X}^{*}, s\left(x^{*}, F\right) \leq s\left(x^{*}, G\right)$ a.s. is obvious. But from Theorem 2.2 in Hiai-Umegaki [20], we have

$$
\begin{aligned}
\mathbf{E}\left(s\left(x^{*}, F\right)\right) & =s\left(x^{*}, \mathbf{E}(F)\right)=s\left(x^{*}, \mathbf{E}(G)\right) \\
& =\mathbf{E}\left(s\left(x^{*}, G\right)\right) .
\end{aligned}
$$


So $s\left(x^{*}, F(\omega)\right)=s\left(x^{*}, G(\omega)\right)$ for all $\omega$ except on a null set $N\left(x^{*}\right)$ related to the point $x^{*}$. Let $N=\bigcup_{x^{*} \in \mathbf{D}^{*}(M)} N\left(x^{*}\right)$; then $\mathbf{P}(N)=0$. Since $\mathbf{D}^{*}(M)$ is dense in the Mackey topology $m\left(\mathbf{X}^{*}, \mathbf{X}\right)$, we have

$$
s\left(x^{*}, F(\omega)\right)=s\left(x^{*}, G(\omega)\right), \omega \in N^{c}, \forall x^{*} \in \mathbf{X}^{*}
$$

Thus $F(\omega)=G(\omega), \omega \in N^{c}$.

The following lemma concerns martingales having three items.

Lemma 3.3. If $\mathcal{F}_{1} \subset \mathcal{F}_{2} \subset \mathcal{F}_{3} \subset \mathcal{F}$ and $\left\{F_{n}, \mathcal{F}_{n}, n=1,2,3\right\}$ is an $\mathcal{L}_{\text {wkc }}^{1}$-valued martingale and there is a sequence $\left\{f^{k}, k \geq 1\right\} \subset S_{F_{3}}^{1}(\mathcal{F})$ such that

$$
\begin{aligned}
& F_{3}=\operatorname{cl}\left\{f^{k} ; k \geq 1\right\}, \text { a.s. } \\
& F_{1}=\operatorname{cl}\left\{\mathbf{E}\left(f^{k} \mid \mathcal{F}_{1}\right) ; k \geq 1\right\}, \text { a.s. },
\end{aligned}
$$

then $F_{2}=\operatorname{cl}\left\{\mathbf{E}\left(f^{k} \mid \mathcal{F}_{2}\right) ; k \geq 1\right\}$, a.s.

Proof. Let $G=\operatorname{cl}\left\{\mathbf{E}\left(f^{k} \mid \mathcal{F}_{2}\right) ; k \geq 1\right\}$. From Theorem 5.1 in Hiai-Umegaki [20] we have

$$
S_{G}^{1}\left(\mathcal{F}_{2}\right) \subset \operatorname{cl}\left\{\mathbf{E}\left(f \mid \mathcal{F}_{2}\right) ; f \in S_{F_{3}}^{1}\left(\mathcal{F}_{3}\right)\right\}=S_{F_{2}}^{1}\left(\mathcal{F}_{2}\right),
$$

where the closure is in the norm of the space $L^{1}(\Omega, \mathbf{X})$. So $G \subset F_{2}$,a.s.

On the other hand,

$$
\begin{aligned}
F_{1} & =\operatorname{cl}\left\{\mathbf{E}\left(\mathbf{E}\left(f^{k} \mid \mathcal{F}_{2}\right) \mid \mathcal{F}_{1}\right) ; k \geq 1\right\} \\
& \subset \mathbf{E}\left(G \mid \mathcal{F}_{1}\right) \subset \mathbf{E}\left(F_{2} \mid \mathcal{F}_{1}\right)=F_{1} \text { a.s. }
\end{aligned}
$$

Therefore $\mathbf{E}\left(G \mid \mathcal{F}_{1}\right)=\mathbf{E}\left(F_{2} \mid \mathcal{F}_{1}\right)$, a.s., thus $\mathbf{E}(G)=\mathbf{E}\left(F_{2}\right)$. By Lemma 3.2, we have $G=F_{2}$, a.s.

Remark 3.1. In Lemma 3.2 and 3.3, we can drop the multifunction set $\mathcal{L}_{w k c}^{1}$ to $\mathcal{L}_{f c}^{1}$ at the expense of introducing the strong separability hypothesis on the dual space $\mathbf{X}^{*}$.

Now we prove the main result of this section.

Theorem 3.2. Let $\left\{F_{t}, \mathcal{F}_{t}, t \in \mathbf{R}_{+}\right\}$be an $\mathcal{L}_{f c}^{1}$-valued martingale. If $\mathbf{X}^{*}$ is strongly separable or $F_{t} \in \mathcal{L}_{w k c}^{1}, \forall t \geq 0$, then there exists a sequence of point-valued martingales $\left\{f_{t}^{k}, \mathcal{F}_{t}, t \in \mathbf{R}_{+}\right\}_{k \geq 1}$ in $M S\left(F_{t}\right)$ such that for all $t \geq 0$ and all $\omega \in \Omega$, $F_{t}(\omega)=c l\left\{f_{t}^{k}(\omega) ; k \in \mathbf{J}\right\}$.

Proof. Using Luu [22, Proposition 1.4] or Hess [17, Corollary 3.3], we can see that for the sampling sequence $\left\{F_{n}, \mathcal{F}_{n}, n \in \overline{\mathbf{J}}\right\}$, there exists a sequence of point-valued martingales $\left\{g_{n}^{k}, \mathcal{F}_{n}, n \in \overline{\mathbf{J}}\right\}_{k \geq 1}$ in $\operatorname{MS}\left(F_{n}\right)$ such that for any $n \in \overline{\mathbf{J}}, F_{n}(\omega)=$ $c l\left\{g_{n}^{k}(\omega) ; k \geq 1\right\}, \forall \omega \in \Omega$.

If $t \in \overline{\mathbf{J}}$, we define $f_{t}^{k}=g_{t}^{k}$ for every $k \geq 1$.

Now for a fixed $t \in \mathbf{R}_{+} \backslash \overline{\mathbf{J}}$, there exists an $n \in \overline{\mathbf{J}}$ such that $n<t<n+1$. Then the three-term martingale $\left\{F_{s}, \mathcal{F}_{s}, s=n, t, n+1\right\}$ satisfies the hypothesis of Lemma 3.3. So we have

$$
F_{t}=\operatorname{cl}\left\{\mathbf{E}\left(f_{n+1}^{k} \mid \mathcal{F}_{t}\right) ; k \geq 1\right\}, \text { a.s. }
$$

where the exceptional set is denoted as $N_{t}, \mathbf{P}\left(N_{t}\right)=0$. Also $F_{t}$ has its Castaing representation $\left(g_{t}^{k}, k \geq 1\right) \subset S_{F_{t}}^{1}\left(\mathcal{F}_{t}\right)$ such that

$$
F_{t}(\omega)=\operatorname{cl}\left\{g_{t}^{k}(\omega), k \geq 1\right\}, \forall \omega \in \Omega .
$$


Defining

$$
f_{t}^{k}(\omega)= \begin{cases}\mathbf{E}\left(f_{n+1}^{k} \mid \mathcal{F}_{t}\right)(\omega), & \omega \in N_{t}^{c}, \\ g_{t}^{k}(\omega), & \omega \in N_{t},\end{cases}
$$

we conclude that $\left(f_{t}^{k}, k \geq 1\right) \subset S_{F_{t}}^{1}\left(\mathcal{F}_{t}\right)$ and, for all $\omega \in \Omega, F_{t}(\omega)=\operatorname{cl}\left\{f_{t}^{k}(\omega) ; k \geq 1\right\}$ and $\left\{f_{t}^{k}, \mathcal{F}_{t}, t \in \mathbf{R}_{+}\right\}$is a point-valued martingale for every $k \geq 1$.

\section{$\S 4$. Convergence Results}

In this section, we give some convergence results to be used in the next section. The first one is a general convergent result concerning an adapted process $\left\{X_{t}, \mathcal{F}_{t}, t \in \mathbf{R}_{+}\right\}$on a metric space $(\mathbf{E}, \rho)$. We can find similar results in Frangos [13] when $\mathbf{E}$ is a Banach space.

Theorem 4.1. Let $\left\{X_{t}, \mathcal{F}_{t}, t \in \mathbf{R}_{+}\right\}$be an $\mathbf{E}$-valued process.

1. If for every bounded stopping time $\tau$, there exists an $\Omega_{\tau}, \mathbf{P}\left(\Omega_{\tau}\right)=1$, such that for any $\omega \in \Omega_{\tau}, \rho-\lim _{s \downarrow \tau, s \in \mathbf{S}} X_{s}(\omega)$ exists, then for almost all $\omega, \rho-$ $\lim _{s \downarrow t, s \in \mathbf{S}} X_{s}(\omega)$ exists for all $t \geq 0$,

2. Suppose that $\left(X_{t}, t \in \mathbf{R}_{+}\right)$has a right limit for every path at $t \geq 0$ and for any bounded predictable time $\tau$ announced by a sequence in $\mathbf{T}(\mathbf{S}), \rho-$ $\lim _{s \uparrow \tau, s \in \mathbf{S}} X_{s}(\omega)$ exists for any $\omega \in \Omega_{\tau}$, where $\mathbf{P}\left(\Omega_{\tau}\right)=1$. Then for almost all $\omega, \rho-\lim _{s \uparrow t, s \in \mathbf{S}} X_{s}(\omega)$ exists for any $t \in \mathbf{R}_{+}$.

The proof of this theorem (but not the statement) is just like that in ChoiSucheston [8, Theorems 2.3 and 3.2]. We need only change $\left\|X_{s}-X_{t}\right\|$ there into $\rho\left(X_{s}, X_{t}\right)$.

Next we turn our attention to the convergence of closed multivalued martingales. Dam [9] and Wang-Xue [34] proved the K-M convergence theorems for right-closed martingales in the ascending case. Recently, Papageorgiou [29] extended the result in Wang-Xue [34] to multivalued uniform amarts.

In the sequel, we discuss discrete parameter processes adapted to an increasing $\sigma$-field filtration $\left(\mathcal{F}_{n}, n \in \mathbf{Z}\right)$. Denote $\mathcal{F}_{\infty}=\sigma\left(\bigcup_{n=1}^{\infty} \mathcal{F}_{n}\right), \mathcal{F}_{-\infty}=\bigcap_{n=1}^{\infty} \mathcal{F}_{-n}$.

Theorem 4.2. Suppose that $F \in \mathcal{L}_{f c}^{1}$ and $F_{n}=\mathbf{E}\left(F \mid \mathcal{F}_{n}\right),-\infty \leq n \leq+\infty$, and suppose either of the following assumptions holds: (a) there exists a $\mathcal{K}$-valued multifunction $G$ such that for all $\omega \in \Omega$ and $1 \leq n \leq+\infty$ we have $F_{n}(\omega) \subset H(\omega)$ (resp. $F_{-n}(\omega) \subset H(\omega)$ ), or (b) $\mathbf{X}^{*}$ is strongly separable. Then $F_{n} \stackrel{\mathrm{K}-\mathrm{M}}{\longrightarrow} F_{\infty}$ a.s. (resp. $F_{-n} \stackrel{\mathrm{K}-\mathrm{M}}{\longrightarrow} F_{-\infty}$ a.s.), as $n \rightarrow \infty$.

Proof. We apply techniques similar to those used in the proof of Papageorgiou [29, Theorem 14] to prove the ascending case. First, by Theorem 3.1, there exists a sequence $\left(f^{i}, i \geq 1\right) \subset S_{F}^{1}$, such that $F(\omega)=c l\left\{f^{i}(\omega), i \geq 1\right\}$ for all $\omega \in \Omega$ and

$$
F_{n}=\operatorname{cl}\left\{\mathbf{E}\left(f^{i} \mid \mathcal{F}_{n}\right) ; i \geq 1\right\}, \quad \text { a.s. }
$$

for all $1 \leq n \leq+\infty$. By the convergence of vector-valued right-closed martingales, for all $i \geq 1$,

$$
\lim _{n \rightarrow \infty}\left\|\mathbf{E}\left(f^{i} \mid \mathcal{F}_{n}\right)-\mathbf{E}\left(f^{i} \mid \mathcal{F}_{\infty}\right)\right\|=0 \quad \text { a.s. }
$$

Thus there exists an $N_{1} \in \mathcal{F}_{\infty}, \mathbf{P}\left(N_{1}\right)=0$, such that for all $\omega \in N_{1}^{c}$,

$$
\lim _{n \rightarrow \infty} \mathbf{E}\left(f^{i} \mid \mathcal{F}_{n}\right)(\omega)=\mathbf{E}\left(f^{i} \mid \mathcal{F}_{\infty}\right)(\omega), \quad i \in \mathbf{J},
$$


and

$$
F_{n}(\omega)=\operatorname{cl}\left\{\mathbf{E}\left(f^{i} \mid \mathcal{F}_{n}\right)(\omega) ; i \in \mathbf{J}\right\}, \forall n \in \mathbf{J}
$$

Hence

$$
F_{\infty}(\omega) \subset s-\underline{\lim }_{n \rightarrow \infty} F_{n}(\omega), \quad \omega \in N_{1}^{c} .
$$

On the other hand, if assumption (a) holds, then from the convergence of rightclosed real-valued martingales, for all $x^{*} \in \mathbf{X}^{*}$

$$
\lim _{n \rightarrow \infty} s\left(x^{*}, F_{n}\right)=s\left(x^{*}, F_{\infty}\right), \quad \text { a.s. }
$$

So there is an $N_{2} \in \mathcal{F}_{\infty}$ satisfying $\mathbf{P}\left(N_{2}\right)=0$ and such that for all $\omega \in N_{2}^{c}$,

$$
\lim _{n \rightarrow \infty} s\left(x^{*}, F_{n}(\omega)\right)=s\left(x^{*}, F_{\infty}(\omega)\right), \quad \forall x^{*} \in \mathbf{D}^{*}(M) .
$$

Hence from Lemma 2.1, we have

$$
F_{n}(\omega) \stackrel{w}{\longrightarrow} F_{\infty}(\omega)(n \rightarrow+\infty) .
$$

This implies that

$$
w-\varlimsup_{n \rightarrow \infty} F_{n}(\omega) \subset F_{\infty}(\omega), \quad \forall \omega \in N_{2}^{c},
$$

by N.S.Papageorgiou [26, Proposition 4.1].

If assumption (b) holds, there is also a null set $N_{3} \in \mathcal{F}_{\infty}$ such that for all $\omega \in N_{3}^{c}$,

$$
\lim _{n \rightarrow \infty} s\left(x^{*}, F_{n}(\omega)\right)=s\left(x^{*}, F_{\infty}(\omega)\right), \quad \forall x^{*} \in \mathbf{D}^{*}
$$

Now for all $1 \leq n \leq+\infty,\left|F_{n}\right| \leq \mathbf{E}\left(|F| \mid \mathcal{F}_{n}\right)$,a.s. Here $\left\{\mathbf{E}\left(|F| \mid \mathcal{F}_{n}\right), \mathcal{F}_{n}, 1 \leq\right.$ $n \leq+\infty\}$ is a right-closed real-valued martingale, so by the Maximal Lemma of supermartingales (cf. C.Dellacherie and P.A.Meyer [11, p.14]), we have

$$
\sup _{1 \leq n \leq \infty}\left|F_{n}\right| \leq \sup _{1 \leq n \leq \infty} \mathbf{E}\left(|F| \mid \mathcal{F}_{n}\right)<\infty \quad \text { a.s. }
$$

Thus there exists another null set $N_{4} \in \mathcal{F}_{\infty}$ including $N_{3}$ such that (4.11) is true for all $\omega \in N_{4}^{c}$. By the usual density narrative, for all $x^{*} \in \mathbf{X}^{*}$ we have

$$
\lim _{n \rightarrow \infty} s\left(x^{*}, F_{n}(\omega)\right)=s\left(x^{*}, F_{\infty}(\omega)\right), \forall \omega \in N_{4}^{c} .
$$

Hence also by Proposition 4.1 in Papageorgiou [26],

$$
w-\overline{\lim }_{n \rightarrow \infty} F_{n}(\omega) \subset F_{\infty}(\omega), \quad \forall \omega \in N_{4}^{c} .
$$

Combining (4.5) and (4.9) (or (4.13)), we obtain the proof.

For the reversed martingales, the proof is similar.

Remark 4.1. Combining Wang-Xue [34, Theorems 2.1 and 3.2], we can also obtain the result for the ascending parameter case.

We have an analogous result for convergence in the Hausdorff metric for reversed martingales with $F \in \mathbf{L}_{c}^{1}$.

Theorem 4.3. Suppose that $F \in \mathbf{L}_{c}^{1}$. Let $F_{-n}=\mathbf{E}\left(F \mid \mathcal{F}_{-n}\right), n \in \mathbf{J}$. Then $F_{-n} \stackrel{h}{\longrightarrow} F_{-\infty}(n \rightarrow \infty)$ a.s. and $\Delta\left(F_{-n}, F_{-\infty}\right) \longrightarrow 0$, where $F_{-\infty}=\mathbf{E}\left(F \mid \mathcal{F}_{-\infty}\right)$. 
Proof. Without loss of generality, we may assume $F$ is $\mathcal{F}_{-1}$-measurable. First suppose that $F_{-1}$ itself is a simple function in $\mathcal{L}_{f c}^{1}$, i.e. $F_{-1}=\sum_{k=1}^{K} H_{k} I_{A_{k}}$, where $\bigcup_{k=1}^{K} A_{k}=\Omega, A_{k} A_{j}=\phi, k \neq j$, and $H_{k} \in \mathcal{C}$. For any $F_{1}, F_{2}, G_{1}, G_{2} \in \mathcal{C}$, it is known that

$$
h\left(F_{1}+F_{2}, G_{1}+G_{2}\right) \leq h\left(F_{1}, G_{1}\right)+h\left(F_{2}, G_{2}\right) .
$$

Thus by the convergence of real reversed martingales

$$
\begin{aligned}
h\left(F_{n}, F_{-\infty}\right) & =h\left(\sum_{k=1}^{K} H_{k} \mathbf{E}\left(I_{A_{k}} \mid \mathcal{F}_{-n}\right), \sum_{k=1}^{K} H_{k} \mathbf{E}\left(I_{A_{k}} \mid \mathcal{F}_{-\infty}\right)\right) \\
& \leq \sum_{k=1}^{K} h\left(H_{k} \mathbf{E}\left(I_{A_{k}} \mid \mathcal{F}_{-n}\right), H_{k} \mathbf{E}\left(I_{A_{k}} \mid \mathcal{F}_{-\infty}\right)\right) \\
& \leq \max _{1 \leq i \leq K}\left|H_{i}\right| \cdot \sum_{k=1}^{K}\left|\mathbf{E}\left(I_{A_{k}} \mid \mathcal{F}_{-n}\right)-\mathbf{E}\left(I_{A_{k}} \mid \mathcal{F}_{-\infty}\right)\right| \\
& \longrightarrow 0 \quad \text { a.s. }(n \longrightarrow \infty) .
\end{aligned}
$$

Now for a general $F_{-1} \in \mathbf{L}_{c}^{1}$ and for any $\epsilon>0$, there is a simple function $H \in \mathcal{L}_{f c}^{1}$ such that $H$ is $\mathcal{F}_{-1}$-measurable and $\Delta\left(F_{-1}, H\right)<\epsilon^{2}$. Assume that $H=\sum_{k=1}^{K} H_{k} I_{A_{k}}$, where $\bigcup_{k=1}^{K} A_{k}=\Omega, A_{k} A_{j}=\phi, k \neq j$, and $H_{k} \in \mathcal{C}$; denote $H_{-n}=\mathbf{E}\left(H \mid \mathcal{F}_{-n}\right), n \geq 1$, and $H_{-\infty}=\mathbf{E}\left(H \mid \mathcal{F}_{-\infty}\right), n \geq 1$. Then by the preceding proof, $h\left(H_{-n}, H_{-\infty}\right) \longrightarrow 0$ a.s. So there exists an $N \in \mathbf{J}$ such that the probability $\mathbf{P}\left(\sup _{n \geq N}\left(h\left(H_{-n}, H_{-\infty}\right)\right)>\epsilon\right)<\epsilon$. For any $n \geq 1$, by Hiai [21, Lemma $2.6]$

$$
\begin{aligned}
h\left(F_{-n}, H_{-n}\right) & =h\left(\mathbf{E}\left(F_{-1} \mid \mathcal{F}_{-n}\right), \mathbf{E}\left(H \mid \mathcal{F}_{-n}\right)\right) \\
& \leq \mathbf{E}\left(h\left(F_{-1}, H\right) \mid \mathcal{F}_{-n}\right):=h_{-n} \\
h\left(F_{-\infty}, H_{-\infty}\right) & \leq \mathbf{E}\left(h\left(F_{-1}, H\right) \mid \mathcal{F}_{-\infty}\right):=h_{-\infty} .
\end{aligned}
$$

Since $\left\{h_{k}, \mathcal{F}_{k},-n \leq k \leq-1\right\}$ is a real martingale for any $n \in \mathbf{J}$, by the Maximal Lemma of supermartingales

$$
\mathbf{P}\left(\max _{-n \leq k \leq-1} h_{k}>\epsilon\right) \leq \frac{1}{\epsilon} \mathbf{E} h_{-1}=\frac{1}{\epsilon} \Delta\left(F_{-1}, H\right)<\epsilon .
$$

Let $n \rightarrow \infty$; we have $\mathbf{P}\left(\sup _{n \geq 1} h_{-n}>\epsilon\right) \leq \epsilon$. We also have

$$
\mathbf{P}\left(h\left(F_{-\infty}, H_{-\infty}\right)>\epsilon\right) \leq \frac{1}{\epsilon} \mathbf{E} h_{-\infty} \leq \frac{1}{\epsilon} \mathbf{E} h_{-1}<\epsilon .
$$

Therefore

$$
\begin{aligned}
\mathbf{P}\left(\sup _{n \geq N} h\left(F_{-n}, F_{-\infty}\right)\right. & >3 \epsilon) \leq \mathbf{P}\left(\sup _{n \geq N} h\left(F_{-n}, H_{-n}\right)>\epsilon\right) \\
& +\mathbf{P}\left(\sup _{n \geq N} h\left(H_{-n}, H_{-\infty}\right)>\epsilon\right) \\
& +\mathbf{P}\left(h\left(H_{-\infty}, F_{-\infty}\right)>\epsilon\right)<3 \epsilon .
\end{aligned}
$$

Hence

$$
F_{-n} \stackrel{h}{\longrightarrow} F_{-\infty}(n \rightarrow \infty) \text { a.s. }
$$


Since $h\left(F_{-n}, F_{-\infty}\right) \leq\left|F_{-n}\right|+\left|F_{-\infty}\right| \leq \mathbf{E}\left(\left|F_{-1}\right| \mid \mathcal{F}_{-n}\right)+\mathbf{E}\left(\left|F_{-1}\right| \mid \mathcal{F}_{-\infty}\right)$, we have

$$
\Delta\left(F_{-n}, F_{-\infty}\right)=\mathbf{E} h\left(F_{-n}, F_{-\infty}\right) \longrightarrow 0(n \longrightarrow \infty)
$$

Remark 4.2. Theorem 4.3 was obtained by Hiai-Umegaki [20, Thm. 6.2] under the additional assumption that $\mathbf{X}$ is reflexive or $F(\omega)$ is compact for a.s. $\omega$. Papageorgiou [29, Thm. 20] proved the convergence of amarts with compact values. Wang-Xue [34, Thm. 3.1] proved the ascending indexed case.

\section{§5. Regularity}

Let $(\mathcal{K}, \rho)$ be the metric space defined in Section 2.

Theorem 5.1. Suppose that $\left\{F_{t}, \mathcal{F}_{t}, t \in \mathbf{R}_{+}\right\}$is an $\mathcal{L}_{w k c}^{1}$-valued martingale. If there exists a multifunction $H$ with weakly compact values such that $F_{t}(\omega) \subset H(\omega)$ for all $\omega \in \Omega$ and $t \geq 0$, then the following assertions are true:

1. For any bounded stopping time $\tau,(\mathrm{K}-\mathrm{M}) \lim _{t \downarrow \tau, t \in \mathbf{S}} F_{t}(\omega)$ exists almost surely (the exceptional null set depends on $\tau$ ).

2. For any bounded predictable time $\tau$ announced by a sequence in $\mathbf{T}(\mathbf{S})$, $(\mathrm{K}-\mathrm{M}) \lim _{t \uparrow \tau, t \in \mathbf{S}} F_{t}(\omega)$ exists almost surely (the exceptional null set depends on $\tau)$.

Proof. (1) We can arbitrarily select a bounded sequence $\left(\tau_{n}, n \in \mathbf{J}\right) \subset \mathbf{T}(\mathbf{S})$ recalling $\tau$. Let $b=\max _{\omega \in \Omega} \tau_{1}(\omega)$. Then $b<\infty$ and $\tau_{n} \leq b$ for $n \geq 1$. Denote $\mathbf{S}_{b}=\{s \in \mathbf{S}: s \leq b\}$; then $\left\{F_{s}, \mathcal{F}_{s}, s \in \mathbf{S}_{b}\right\}$ is an $\mathcal{L}_{w k c}^{1}$-valued martingale right closed by $F_{b}$. This is also true for $\left\{F_{\tau_{n}}, \mathcal{F}_{\tau_{n}}, n \in \mathbf{J}\right\}$ by Papageorgiou [29, Theorem 12]. So by Theorem 4.2 , we have

$$
(\mathrm{K}-\mathrm{M}) \lim _{n \rightarrow \infty} F_{\tau_{n}}=G:=\mathbf{E}\left(F_{b} \mid \mathcal{F}_{\tau+}\right)=\mathbf{E}\left(F_{b} \mid \mathcal{F}_{\tau}\right), \quad \text { a.s. }
$$

the exceptional null set depends on $\tau$. Let

$$
\xi_{t}(\omega)= \begin{cases}\rho\left(F_{t}(\omega), G(\omega)\right), & t>\tau(\omega), \\ 0, & t \leq \tau(\omega) .\end{cases}
$$

Then $\left\{\xi_{t}, \mathcal{F}_{t}, t \in \mathbf{R}_{+}\right\}$is an adapted process and

$$
\lim _{n \rightarrow \infty} \xi_{\tau_{n}}=0, \quad \text { a.s. }
$$

Since the sequence $\left(\tau_{n}\right)$ is chosen arbitrarily, we know by Frangos [13, Theorem 2.2 ] that

$$
\lim _{t \downarrow \tau, t \in \mathbf{S}} \xi_{t}=0, \quad \text { a.s.; }
$$

the exceptional null set depends on $\tau$.

(2) Similarly, for a fixed bounded predictable time $\tau$ announced by any sequence $\left(\tau_{n}\right) \subset \mathbf{T}(\mathbf{S})$, we can prove that

$$
(\mathrm{K}-\mathrm{M}) \lim _{n \rightarrow \infty} F_{\tau_{n}}=G_{1}:=\mathbf{E}\left(F_{b_{1}} \mid \mathcal{F}_{\tau-}\right), \quad \text { a.s. }
$$

where $b_{1}=\sup _{\omega \in \Omega} \tau(\omega)<\infty$ and the exceptional null set depends on $\tau$ and $G_{1} \in \mathcal{L}_{w k c}^{1}$ by Papageorgiou [29, Proposition 3]. This means that for any fixed 
$x \in \mathbf{D}$ and $x^{*} \in \mathbf{D}^{*}(M)$

(a) $\lim _{n \rightarrow \infty} d\left(x, F_{\tau_{n}}\right)=d\left(x, G_{1}\right)$, a.s.,

(b) $\lim _{n \rightarrow \infty} s\left(x^{*}, F_{\tau_{n}}\right)=s\left(x^{*}, G_{1}\right)$, a.s.

Also from Frangos [13, Thm. 2.2], there exist two exceptional null sets $N(x)$ and $N\left(x^{*}\right)$ s.t.

$\left(\mathrm{a}^{\prime}\right) \lim _{t \uparrow \tau(\omega), t \in \mathbf{S}} d\left(x, F_{\tau_{n}}(\omega)\right)=d\left(x, G_{1}(\omega)\right), \quad \omega \in N(x)^{c}$,

$\left(\mathrm{b}^{\prime}\right) \lim _{t \uparrow \tau(\omega), t \in \mathbf{S}} s\left(x^{*}, F_{\tau_{n}}(\omega)\right)=s\left(x^{*}, G_{1}(\omega)\right), \quad \omega \in N\left(x^{*}\right)^{c}$.

Let $N=\left(\bigcup_{x \in \mathbf{D}} N(x)\right) \cup\left(\bigcup_{x^{*} \in \mathbf{D}^{*}(M)} N\left(x^{*}\right)\right) \in \mathcal{F}_{\tau_{-}}$. Then $\mathbf{P}(N)=0$, and $N$ only depends on $\tau$. By Lemma 2.2

$$
(\mathrm{K}-\mathrm{M}) \lim _{t \uparrow \tau(\omega), t \in \mathbf{S}} F_{t}(\omega)=G_{1}(\omega), \quad \omega \in N^{c} .
$$

We obtain the proof.

The above result and Theorem 4.1 imply the following theorem.

Theorem 5.2. If the hypotheses of Theorem 5.1 hold, then for almost all $\omega \in \Omega$

1. $\forall t \geq 0, \quad(\mathrm{~K}-\mathrm{M}) \lim _{s \downarrow t, s \in \mathbf{S}} F_{s}(\omega)$ exists,

2. $\forall t>0, \quad(\mathrm{~K}-\mathrm{M}) \lim _{s \uparrow t, s \in \mathbf{S}} F_{s}(\omega)$ exists.

Now we prove one of the main results of this section.

Theorem 5.3. If the hypotheses of Theorem 5.1 hold, then there exists an adapted process $\left\{\tilde{F}_{t}, \mathcal{F}_{t}, t \in \mathbf{R}_{+}\right\}$with values in $\mathcal{L}_{w k c}^{1}$ such that

1. $\left(\tilde{F}_{t}, t \in \mathbf{R}_{+}\right)$is $K$-M right continuous and, for almost all $\omega$,

$$
\tilde{F}_{t}(\omega)=(\mathrm{K}-\mathrm{M}) \lim _{s \downarrow t, s \in \mathbf{S}} F_{s}(\omega), \quad t \geq 0,
$$

2. for almost all $\omega, \widetilde{F}_{t-}(\omega)=(\mathrm{K}-\mathrm{M}) \lim _{s \uparrow t, s \in \mathbf{R}_{+}} \widetilde{F}_{s}(\omega)$ exists for every $t>0$ and $\tilde{F}_{t-}(\omega)=(\mathrm{K}-\mathrm{M}) \lim _{s \uparrow t, s \in \mathbf{S}} F_{s}(\omega)$,

3. $\forall t \geq 0, \quad \tilde{F}_{t}=F_{t}$, a.s.,

4. $\left\{\tilde{F}_{t}, \mathcal{F}_{t}, t \in \mathbf{R}_{+}\right\}$is a multivalued martingale.

Proof. (1) For $N$ the exceptional null set in Theorem 5.2, we define

$$
\tilde{F}_{t}(\omega)= \begin{cases}(\mathrm{K}-\mathrm{M}) \lim _{s \downarrow t, s \in \mathbf{S}} F_{s}(\omega), & \omega \in N^{c}, \\ \{\mathbf{0}\}, & \omega \in N ;\end{cases}
$$

then $\left\{\tilde{F}_{t}, \mathcal{F}_{t}, t \in \mathbf{R}_{+}\right\}$is an $\mathcal{L}_{w k c}^{1}$-valued adapted process. For a fixed $t \geq 0$, when $\omega \in N, \tilde{F}$. $(\omega)$ is apparently K-M right continuous. Now for $\omega \notin N, \forall \epsilon>0$, there exists a $\delta>0$, such that for any $s \in \mathbf{S}$ satisfying $0<s-t<\delta, \rho\left(\tilde{F}_{t}(\omega), F_{s}(\omega)\right)<\epsilon$. Thus for $r>t$ satisfying $r-t<\delta$, we have

$$
\rho\left(\tilde{F}_{t}(\omega), \tilde{F}_{r}(\omega)\right)=\lim _{s \downarrow r, s \in \mathbf{S}} \rho\left(\tilde{F}_{t}(\omega), F_{s}(\omega)\right) \leq \epsilon .
$$

Hence $\tilde{F}_{.}(\omega)$ is K-M right continuous.

(2) For $t>0, \omega \in N^{c},(\mathrm{~K}-\mathrm{M}) \lim _{s \uparrow t, s \in \mathbf{S}} F_{s}(\omega)$ exists. The rest of the proof is similar to (1). 
(3) $\forall t \geq 0$, by Theorem 4.2

$$
\tilde{F}_{t}=\mathbf{E}\left(F_{t+1} \mid \mathcal{F}_{t+}\right)=\mathbf{E}\left(F_{t+1} \mid \mathcal{F}_{t}\right)=F_{t}, \quad \text { a.s. }
$$

(4) This is apparently true from (3).

Using Theorem 3.3, Wang-Xue [34, Thm. 3.1], and the similar proofs of Theorem 5.1 to Theorem 5.3, we have the following regularity result for multivalued martingales with $\mathbf{L}_{c}^{1}$ values in convergence in the Hausdorff metric.

Theorem 5.4. Suppose that $\left\{F_{t}, \mathcal{F}_{t}, t \in \mathbf{R}_{+}\right\}$is an $\mathbf{L}_{c}^{1}$-valued martingale. Then there exists an adapted process $\left\{\widetilde{F}_{t}, \mathcal{F}_{t}, t \in \mathbf{R}_{+}\right\}$with values in $\mathbf{L}_{c}^{1}$ such that

1. $\left(\tilde{F}_{t}, t \in \mathbf{R}_{+}\right)$is Hausdorff right-continuous and, for almost all $\omega$,

$$
\tilde{F}_{t}(\omega)=h-\lim _{s \uparrow t, s \in \mathbf{S}} F_{s}(\omega), \quad t \geq 0,
$$

2. for almost all $\omega, \widetilde{F}_{t-}(\omega)=h-\lim _{s \uparrow t, s \in \mathbf{R}_{+}} \widetilde{F}_{s}(\omega)$ exists for every $t>0$ and $\tilde{F}_{t-}(\omega)=h-\lim _{s \uparrow t, s \in \mathbf{S}} F_{s}(\omega)$,

3. $\forall t \geq 0, \quad \tilde{F}_{t}=F_{t}$, a.s.,

4. $\left\{\tilde{F}_{t}, \mathcal{F}_{t}, t \in \mathbf{R}_{+}\right\}$is a multivalued martingale.

\section{ACKNOWLEDGEMENTS}

The authors wish to thank the referee for very helpful comments on the original version. Also the authors wish to thank Professor R. Durrett for his kind and helpful attitude during the review process.

\section{REFERENCES}

1. S.Bagchi, On a.s. convergence of classes of multivalued asymptotic martingales, Ann. Inst. Henri Poincaré 21 (1985), 313-321. MR 87f:60009

2. S.Bagchi, Characterisations of classes of multivalued processes using Riesz approximations, J.Multivariate Anal. 42 (1992), 23-34. MR 93h:60072

3. G.Beer, Convergence of continuous linear functionals and their level sets, Arch. Math. 52, (1989), 482-491. MR 90i:46018

4. G.Beer, Support and distance functionals for convex sets, Number. Funct. Anal. and Optimiz. 10 (1989), 15-36. MR 89m:46031

5. C.Castaing,M.Valadier, Convex analysis and measurable multifunctions, Lecture Notes in Math., vol. 580, Springer-Verlag, Berlin, 1977. MR 57:7169

6. R.V.Chacon,L.Sucheston, On convergence of vector valued asymptotic martingales, Z. Wahrs. verw. Gebiete 33 (1975), 55-59. MR 52:15658

7. S.Chatterji, Vector values martingales and their applications, Lecture Notes in Math., vol. 526, Spring- er-Verlag, Berlin and New York, 1976, pp. 33-51. MR 58:24529

8. B.Choi,L.Sucheston, Continuous parameter uniform amarts, Lecture Notes in Math., vol. 860, Sprin- ger-Verlag, Berlin, 1981, pp. 85-98. MR 83f:60075

9. B.K.Dam, Almost sure convergence of set-valued martingales and submartingales., Acta Math. Hung. 60 (1992), 197-205. MR 94h:60070

10. J.P.Daures, Version multivoque du théoréme de Doob, Ann. Inst. Henri Poincaré 9 (1973), 167-176. MR 51:14256

11. C.Dellacherie,P.A.Meyer, Probabilities and potentials B,Chap. VI, North-Holland, Netherlands, 1982. MR 85e:60001

12. L.Egghe, Strong convergence of pramarts in Banach spaces, Cand. J. Math. 33 (1981), 357361. MR 82m:60052

13. N.E.Frangos, On regularity of Banach-valued processes, Ann. Prob. 13 (3) (1985), 985-990. MR 86k:60011 
14. N.E.Frangos, On convergence of vector valued pramarts and subpramarts, Canad. J. Math. 37 (1985), 260-270. MR 86h:60097

15. E.Giné,M.G.Hahn and J.Zinn, Limit theorems for random sets:An application of probability in Banach space results, Lecture Notes in Math., vol. 990, Springer-Verlag, Berlin and New York, 1983, pp. 112-135. MR 85d:60019

16. A.Gut,K.D.Schmidt, Amarts and set function processes, Lecture Notes in Math. vol. 1042 Springer- Verlag, Berlin and New York, 1983. MR 85k:60064

17. C.Hess, On multivalued martingales whose values may be unbounded:martingale selectors and Mosco convergence, J.Multivariate Anal. 39 (1991), 175-201. MR 93e:60090

18. C.Hess, Convergence of conditional expectations for unbounded random sets, integrals, and integrals functionals, Math. Oper. Res. 16 (3) (1991(August)), 627-649. MR 92h:49012

19. C.Hess, Existence of martingale selectors and convergence of multivalued supermartingales, C.R.Acad. Sci. Paris Sér. I Math. 312 (1) (1991), 149-154. MR 91m:60083

20. F.Hiai,H.Umegaki, Integrals, conditional expectations, and martingales of multivalued functions, J.Mul- tivariate Anal. 7 (1977), 149-182. MR 58:22463

21. F.Hiai, Convergence of conditional expectations and strong laws of large numbers for multivalued random variables, Trans. Ameri. Math. Soc. 291 (2) (1985), 613-627. MR 86k:60048

22. D.Q.Luu, Representations and regularity of multivalued martingales, C.R.Acad. Sci. Paris, Serie 1 300, (2) (1985), 63-65. MR 86h:60096

23. A.Millet,L.Sucheston, Characterizations of Vitali Conditions with overlap in terms of convergence of amarts, Canad. J. Math. 31 (1979), 1033-1046. MR 80j:60080

24. A.Millet,L.Sucheston, Convergence of classes of amarts indexed by directed sets, Canad. J. Math. 32 (1980), 86-125. MR 81g:60051

25. N.S.Papageorgiou, On the theory of Banach space valued multifunctions,1.Integration and conditional expectation, J.Multivariate Anal. 17 (1985), 185-206. MR 87e:28015

26. N.S.Papageorgiou, Convergence theorems for Banach space valued integrable multifunctions, Inter. J.Math. \& Math. Sci. 10, (1987), 433-442. MR 88i:28019

27. N.S.Papageorgiou, Convergence and representation theorems for set-valued random processes, Stoch. Anal. Appl. 7 (1989), 187-210. MR 91a:60032

28. N.S.Papageorgiou, Convergence and representation theorems for set-valued random processes, J.Math. Anal. Appl. 150 (1990), 129-145. MR 92a:60117

29. N.S.Papageorgiou, On the conditional expectation and convergence properties of random sets, Trans. Amer. Math. Soc. 347 (7) (1995), 2495-2515. MR 95i:60037

30. G.Salinetti,R.Wets, On the relations between two types of convergence for convex functions, J. Math. Anal. Appl. 60 (1977), 211-226; errata, 66 (1978), 689. MR 57:18828; MR 80a:90114

31. G.Salinetti,R.Wets, On the convergence of closed-valued measurable multifunctions, Trans. Amer. Math. Soc. 266 (1981), 275-289. MR 82k:28007

32. M.Slaby, Strong convergence of vector-valued pramarts and subpramarts, Prob. Math. Statist. 5 (1985), 187-190. MR 87c:60042

33. M.Tsukada, Convergence of best approximations in a smooth Banach space, J. Approx. Th. 40 (1984), 301-309. MR 86a:41034

34. Z.P.Wang,X.H.Xue, On convergence and closedness of multivalued martingales, Trans. Amer. Math. Soc. 341 (2) (1994), 807-827. MR 94d:60074

Department of Mathematics, University of Southern California, Los Angeles, CalIFORNIA 90089

E-mail address: wdong@scf .usc.edu

Department of Mathematical Statistics, East China Normal University, Shanghai 200062, People's Republic of China 